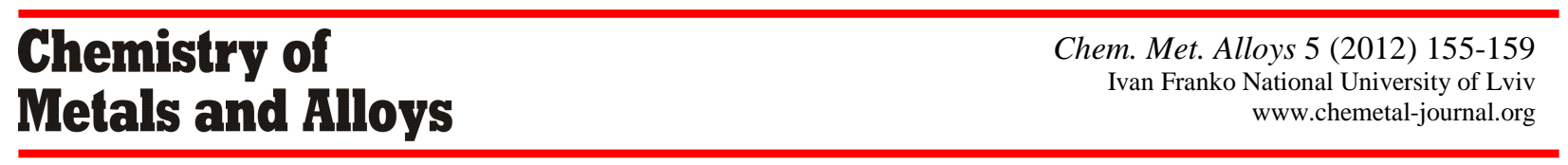

\title{
Dominant point defects in germanium telluride crystals
}

\author{
Dmytro FREIK ${ }^{1}$, Igor GORICHOK ${ }^{1}$, Liubov YURCHYSHYN ${ }^{1}$ \\ ${ }^{1}$ Institute of Physics and Chemistry, Precarpathian National Vasyl Stefanyk University, \\ Shevchenka St. 57, 76018 Ivano-Frankivsk, Ukraine \\ * Corresponding author.Tel.: +380-34-2596082; e-mail: goritchok@rambler.ru
}

Received December 10, 2012; accepted December 26, 2012; available on-line July 5, 2013

The features of the experimental dependences of the concentration of charge carriers on the temperature and chemical composition of germanium telluride with NaCl-type structure at temperatures $T=550-850 \mathrm{~K}$ and concentrations of excess tellurium $X_{T e}=\mathbf{0 . 0 1 - 0 . 1}$ at.\% Te are interpreted, and a crystal chemical model is proposed for the defect subsystem. It was found that the dominant defects under these conditions are doubly ionized metal vacancies, which define the character of the dependences $p(T), p\left(X_{T e}\right)$. At temperatures above $750 \mathrm{~K}$ and for excess tellurium concentrations above 0.04 at. \% Te, also antistructural chalcogen atoms have a significant impact on the concentration of free holes. The concentrations of other defects are much lower and do not affect the electrical properties of the material.

Germanium telluride / Electrical properties / Point defects

\section{Introduction}

Semiconductor compounds IV-VI and solid solutions based on them are basic materials for modern infrared electronics and are used to create injection heterolasers, LEDs and photodetectors. An important advantage of devices based on them is the possibility of restructuring the spectral characteristics caused by changes in composition, temperature, or pressure, due to the dependence of the band gap on these parameters [1]. Germanium telluride is in addition a high-performance thermoelectric material in a range of temperatures from room temperature to (800-900) $\mathrm{K}$ [1].

GeTe is a degenerate semiconductor compound, which is characterized by structural instability. The process of crystallization of $(\beta-\mathrm{GeTe})$ in a $\mathrm{NaCl}$-type structure at temperatures above $650 \mathrm{~K}$ takes place with a significant deviation from stoichiometry toward excess tellurium, which leads to exclusively $p$-type conductivity [2]. When the temperature decreases to values of (600-650) $\mathrm{K}$ the $\alpha$-modification of GeTe with a rhombohedral lattice is stable. With excess tellurium another lowtemperature, orthorhombic modification of germanium telluride $(\gamma$-GeTe) occurs.

Despite the large number of papers devoted to the peculiarities of the properties of germanium telluride, the defect subsystem of the material is not enough explored. In particular, the question of the effect of antistructural defects on the electrical properties is controversial [3-7]. In most studies, the conclusions on the predominant type of defects are made based on indirect experimental measurements, which are not always unambiguously interpreted. One way to identify the point defects can be simulation of results using the method of thermodynamic potential efficiency, as confirmed in [8,9]. This approach has several advantages over the traditionally used method of Kröger's quasichemistry reactions [10], because it can properly consider degenerate carrier statistics. On the basis of minimization of the free energy we performed an investigation of the defect subsystem in $\beta$-GeTe crystals, which includes doubly ionized vacancies $V_{G e}^{2-}$, singly ionized vacancies of tellurium $V_{T e}^{1+}$, and antistructural atoms of both types $\left(T e_{G e}^{1+}, G e_{T e}^{1-}\right)$.

\section{Method of calculating the concentration of defects}

The concentration of point defects in $\beta$-GeTe at a temperature $T$ and for a given excess of tellurium $X_{T e}$ can be determined by minimizing the free energy of the crystal $[11,12]$ :

$$
F=F_{0}+\sum E[D]+n E_{C}-p E_{V}-T\left(S_{k}+S_{n}+S_{p}\right)
$$

taking into account the condition:

$\left[V_{G e}^{2-}\right]+\left[V_{T e}^{+}\right]+2\left[G e_{T e}^{-}\right]+2\left[T e_{G e}^{+}\right]=X_{T e}$. 
Here $F_{0}$ - free energy that does not depend on the presence of defects, $E$ - energy of formation of point defects, $[D]$ - concentration of defects $D, n$ and $p-$ concentration of electrons and holes, $E_{C}$ and $E_{V}-$ the energy of the bottom of the conduction band and the upper edge of the valence band, $S_{k}$ - configuration entropy, $S_{n}$ and $S_{p}$ - entropy of electrons in the conduction band and holes in the valence band. The summation is over all sublattices and all defects in the sublattice.

Entropy is determined by the Boltzmann law:

$$
S=k \ln \left(\Pi W_{j}\right) \text {, }
$$

where $W_{j}$ - thermodynamic probability of the $j$-th sublattice for configuration entropy, or $j$-th allowed band for the entropy of electrons and holes. For a sublattice with several different types of defect:

$$
W_{j}=\frac{N_{J} !}{\left(N_{J}-\sum[D]\right) ! \cdot \Pi[D] !},
$$

where $N_{J}$ - concentration of nodes where defects can form.

For electrons and holes the thermodynamic probability is:

$$
W_{n}=\frac{N_{C} !}{\left(N_{C}-n\right) ! n !}, W_{p}=\frac{N_{V} !}{\left(N_{V}-p\right) ! p !},
$$

where $N_{C}$ and $N_{V}$ - density of states in the conduction band and valence band, respectively.

Using the data of [13] on the concentration dependence of the effective mass of holes in GeTe, and assuming the validity of the same dependence for electrons, their concentration can be calculated by the formulae:

$$
n=N_{C} \cdot a \mathrm{e}^{b \frac{\mu}{k T}}, p=N_{V} \cdot a \mathrm{e}^{-b \frac{\mu+E_{g}}{k T}} \text {. }
$$

Here

$$
N_{C}=a \cdot N_{C, 0}^{2} \cdot \alpha^{3} \cdot \mathrm{e}^{b \frac{\mu}{k T}}, N_{C, 0}=\left(\frac{2 \pi m_{e, 0}^{*} k T}{h^{2}}\right)^{\frac{3}{2}}
$$

and

$$
N_{V}=a \cdot N_{V, 0}^{2} \cdot \alpha^{3} \cdot \mathrm{e}^{-b \frac{\mu+E_{g}}{k T}}, N_{V, 0}=\left(\frac{2 \pi m_{h, 0}^{*} k T}{h^{2}}\right)^{\frac{3}{2}} \text {, }
$$

where the coefficients $a$ and $b$ are amendments that take into account the degeneracy of carriers and are calculated by approximation of the Fermi integral, $E_{g}$ - band gap.

The chemical potential of electrons $\mu$ was determined from the equation of electroneutrality:

$$
\sum Z[D]=n-p
$$

where:

$$
\mu=\frac{1}{2 b} \times k T \ln \left(\frac{\sum Z[D]+\sqrt{\left(\sum Z D\right)^{2}+4 \alpha^{6} a^{2} N_{C, 0}^{2} \cdot N_{V, 0, l}^{2} \mathrm{e}^{-2 b \frac{E_{g, l}}{k T}}}}{2 \alpha^{3} a^{2} N_{C, 0}^{2}}\right)
$$

where $Z$ - charge of the defect.
In formulae (6-8), similarly to the works of [8,9], the experimental dependences of $m^{*}(p)$ in [13] were approximated by the function:

$$
\frac{m^{*}}{m_{0}}=\alpha p^{\beta}=0,2 \cdot 10^{-6} \cdot p^{\frac{1}{3}} \text {. }
$$

The minimum of free energy (1) was found numerically by random perturbations, and the initial (starting) value of the coordinate set randomly. Step perturbations varied randomly from zero to some maximum value. With increasing number of iterations the maximum step of disturbance decreased by an exponential law. Terms in (2) were taken into account by the method of penalty functions.

\section{Results and discussion}

The results of the calculation of the concentration of point defects depending on the temperature for an excess of 0.015 at.\% tellurium are presented in Fig. 1. The experimental values were obtained using the temperature dependence of the Hall coefficient from [4] and the temperature dependence of the Hall factor from [13]. It is seen that the concentrations of vacancies of tellurium $V_{T e}^{1+}$ and antistructural germanium atoms $G e_{T e}^{1-}$ are much smaller than the concentrations of germanium vacancies $V_{G e}^{2-}$ and antistructural tellurium atoms $T e_{G e}^{1+}$. Given this result, it is reasonable to imagine a model in which only $V_{G e}^{2-}$ and $T e_{G e}^{1+}$ are considered, as in this case it is possible to obtain the analytical solution of (1), (2) and, therefore, more accurate calculated values of the concentrations of point defects [D].

Thus, differentiating equation (1) on the vacancy concentration of germanium $V_{G e}^{2-}$ and setting the resulting expression to zero, in view of (2), we obtain:

$V_{G e}^{2-}=\frac{1}{15}\left(A+\frac{X_{T e}^{2}}{A}+X_{T e}\right),\left[T e_{G e}^{+}\right]=\frac{X_{T e}-\left[V_{G e}^{2-}\right]}{2}$,

where

$$
\begin{aligned}
& A=\left(675 E^{2}+X_{T e}^{3}+15 \sqrt{2025 E^{4}+6 E^{2} X_{T e}^{3}}\right)^{\frac{1}{3}}, \\
& E=N_{c a t}\left(\frac{K^{*}}{K^{1 / 2}}\right) \exp \left(-\frac{E_{V_{G e}^{2-}}+2 E_{V}+b E_{g}}{k T}+1\right), \\
& K=\alpha^{3} \cdot N_{V, 0}^{2} \cdot a^{2}, K^{*}=\alpha^{3} \cdot N_{V, 0}^{2} \cdot a \cdot \mathrm{e}^{-b \frac{E_{g}}{k T}} .
\end{aligned}
$$

Fig. 2 presents the experimental values and theoretical values calculated by formulas (12)-(14) for the dependence of the hole concentration on the temperature with an excess of tellurium of 0.015 at.\% (curve 1) and 0.04 at.\% (curve 2). The qualitative difference between these relationships are due to peculiarities of the formation of the defect subsystem 


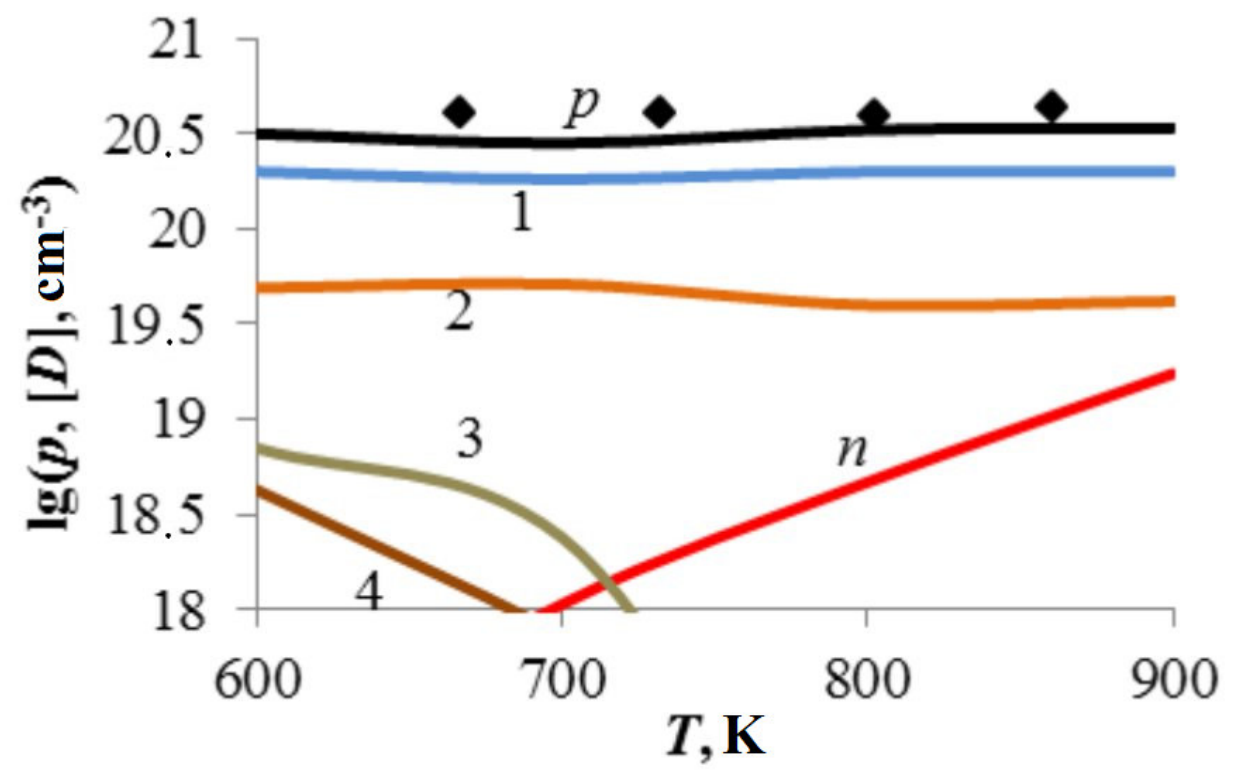

Fig. 1. Dependence of the concentrations of holes $p$, electrons $n$ and point defects $\left(1-\left[V_{G e}^{2-}\right], 2-\left[T e_{G e}^{1+}\right]\right.$, $\left.3-\left[V_{T e}^{1+}\right], 4-\left[G \mathrm{e}_{T e}^{1-}\right]\right)$ in $\beta-G e T e$ crystals on temperature. Curves - numerical calculation by minimization of the free energy of the crystal, - experiment [4]. The concentration of excess tellurium is 0.015 at. $\%$.

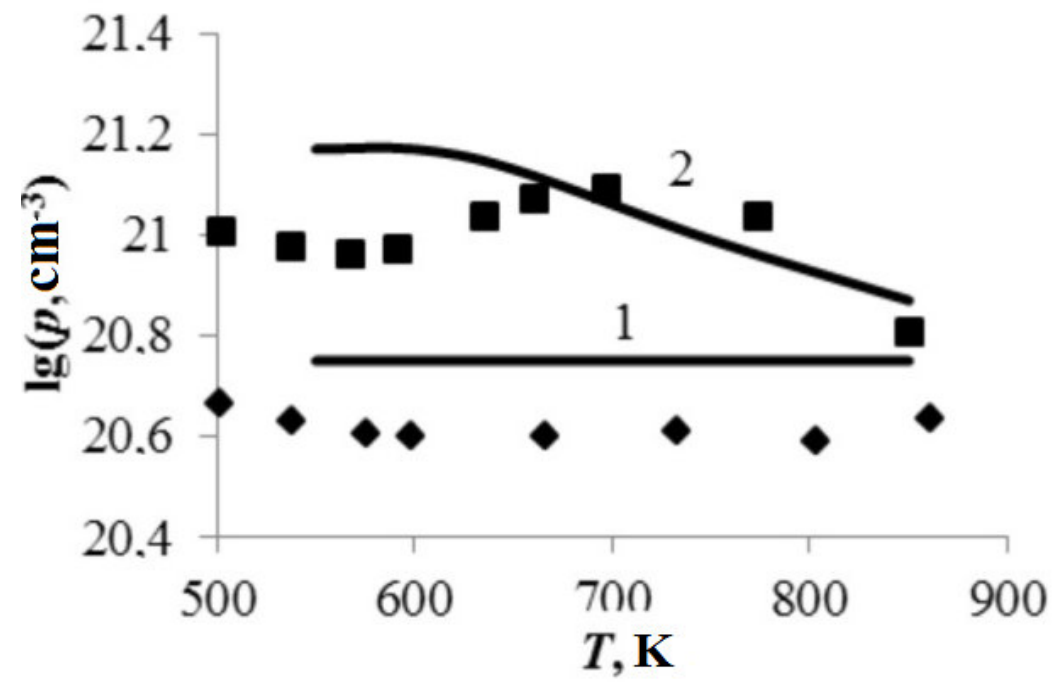

Fig. 2 Dependence of the hole concentration $p$ in $\beta$-GeTe crystals on temperature for two concentrations of excess tellurium: $1(\diamond)-X_{T e}=0.015$ at.\% Te, $2(\boldsymbol{\square})-X_{T e}=0.04$ at.\% Te. Curves - analytical calculations, $\mathbf{n},-$ experiment [4].

of the material: at low concentrations of excess tellurium (0.015 at.\%) the dominant defects are doubly ionized vacancies of germanium $V_{G e}^{2-}$ and antistructural tellurium atoms $T e_{G e}^{1+}$ do not affect the concentration of holes that was previously obtained by numerical minimization (1); elevated concentrations of excess Te lead to an increase of the concentration of antistructural defects (Fig. 3), which also causes a decline of the hole concentration (Figs. 2 and 3).

Based on the obtained results, it can be argued that the dominance of antistructural tellurium atoms $T e_{G e}^{1+}$ in the high-temperature $\beta$-modification of GeTe is unlikely. The decrease of the concentration with decreasing temperature may 


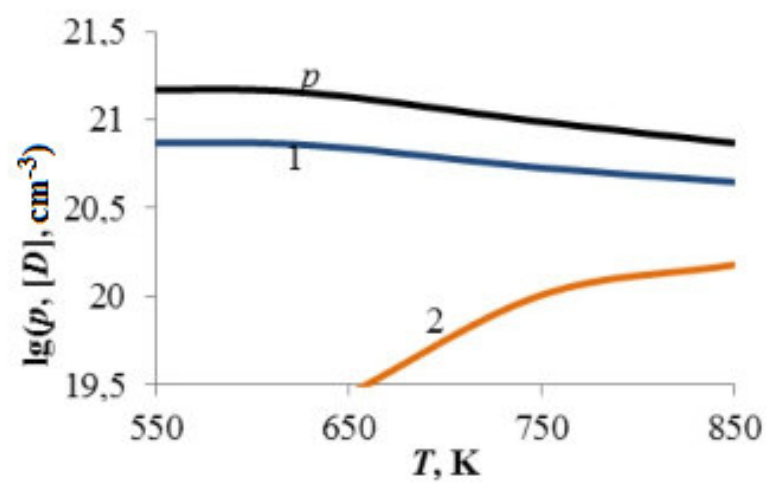

(a)

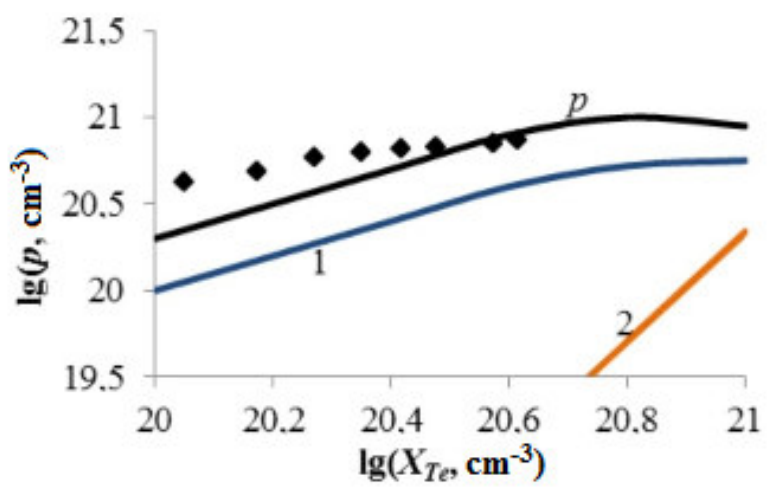

(b)

Fig. 3 Dependence of the hole concentration $p$ and concentrations of point defects $\left(1-\left[V_{G e}^{2-}\right], 2-\left[T e_{G e}^{1+}\right]\right)$ in $\beta$-GeTe crystals on temperature (a) and the concentration of excess tellurium (b). Curves - analytical calculations, - experiment [14].

also indicate that in the $\alpha$-phase these defects are not predominant. However, the differences in the energy structure of the $\alpha$ - and $\beta$-phases can also lead to significant differences in the behavior of the point defects. Dominance of antistructural germanium atoms, which is characteristic for crystals with significant excess chalcogen, can be predicted for the orthorhombic modification of germanium telluride ( $\gamma$-phase).

It should be noted that some differences in the numerical values of the theoretically calculated dependences $p\left(T, X_{T e}\right)$ with respect to the experimental values are due to the complexity of determining the energy parameters of point defects. In particular, the ionization energy of germanium vacancies is known only very approximately (0.1-0.2 eV below the upper edge of the valence band $[2,15])$. Since the Fermi level, according to the calculated data, is very close to the level of germanium vacancies $(0.05-0.1 \mathrm{eV}$ below the upper edge of the valence band), the accuracy of its determination greatly affects the result of the calculations. Despite this, the theoretical dependence accurately describes the Hall measurement data, which should be an indication for the adequacy of the model proposed for the defect subsystem.

\section{Conclusions}

1. It was found that in the high-temperature modification $\beta$-GeTe the dominant defects are doubly ionized germanium vacancies $V_{G e}^{2-}$, which determine the hole concentration dependence on the temperature and chemical composition of the crystal.

2. It was shown that the concentration of antistructural tellurium atoms $\left[T e_{G e}^{1+}\right]$ is an order of magnitude lower than the concentration of germanium vacancies $\left[V_{G e}^{2-}\right]$ and their influence on the concentration of charge carriers is noticeable at temperatures above $750 \mathrm{~K}$ and excess tellurium concentrations above 0.04 at.\% Te. Dominance of $T e_{G e}^{1+}$ can be predicted only for the $\gamma$-modification of germanium telluride.

3. It was found that tellurium vacancies $V_{T e}^{1+}$ and antistructural germanium atoms $G e_{T e}^{1-}$ under any of the studied conditions did not affect the concentration of free holes, and their concentrations are several orders of magnitude lower than the concentrations $\left[V_{G e}^{2-}\right]$ and $\left[T e_{G e}^{1+}\right]$.

4. The theoretically calculated concentrations of charge carriers in GeTe crystals $p(T)$ and $p\left(X_{T e}\right)$ are in satisfactory agreement with the Hall measurements, indicating the validity of the conclusions regarding the dominant type of defects.

\section{References}

[1] D.S. Bletskan, Crystalline and Glassy Chalcogenides, Si, Ge, Sn and Their Alloys. Vol. 1, Zakarpattya, Uzhgorod, 2004, 290 p.

[2] Ye.P. Sabo, J. Thermoelectr. (3) (2000) 30-46.

[3] B.F. Hruzinov, T.B. Zhukova, N.V. Kolomoiets, P.P. Konstantinov, E.Ya. Lev, L.M. Sysoieva, Izv. Akad. Nauk, Neorg. Mater. 10(7) (1974) 1219-1225.

[4] B.F. Hruzinov, P.P. Konstantinov, B.Ya. Moizhes, Yu.I. Ravich, L.M. Sysoieva, Fiz. Tekh. Poluprovodn. 10(3) (1976) 497-502.

[5] I.A. Chernik, P.P. Konstantinov, A.H. Vyshynskii, A.V. Berezin, Phys. Technol. Semicond. 28 (1986) 1939-1941. 
[6] I.A. Chernik, A.H. Vyshynskii, A.V. Berezin, S.N. Lykov, Fiz. Tekh. Poluprovodn. 21 (1987) 1261-1266.

[7] O.Sh. Hohishvili, V.S. Kononykhin, S.P. Lalykin, Zh. Neorg. Khim. 24 (1979) 860-864.

[8] D. Freik, I. Gorichok, Yu. Lysiuk, M. Shevchuk, Chem. Met. Alloys 5(1/2) (2012) 1-7.

[9] D.M. Freik, I.V. Gorichok, M.O. Shevchuk, Ukr. Khim. Zh. 78(9) (2012) 25-29.

[10] F. Kröger, Chemistry of Imperfect Crystals, Mir, Moscow, 1972, 640 p. (in Russian).

[11] I. Bolesta, Solid State Physics, Publ. Center Ivan Franko National University of Lviv, 2003, 480 p. (in Ukrainian).
[12] S.V. Buliarskii, V.V. Svietukhin, P.E. Lvov, Fiz. Tekh. Poluprovodn. 34 (2000) 385-388.

[13] M.A. Korzhuiev, Fiz. Tverd. Tela 35 (1993) 3043-3052.

[14] N.Kh. Abrikosov, L.V. Poretskaia, Ye.V. Surovtseva, L.Ye. Shelimova, Semiconductor Compounds, Their Preparation and Properties, Nauka, Moscow, 1967, 177 p. (in Russian).

[15] A. Edwards, Theory of Intrinsic Defects in Crystalline GeTe and of Their Role in Free Carrier Transport, Final Report, Air Force Research Laboratory, Kirtland, 2008, 10 p. 